\title{
Comparative genomic analysis of eutherian fibroblast growth factor genes
}

\author{
Marko Premzl|D
}

\begin{abstract}
Background: The eutherian fibroblast growth factors were implicated as key regulators in developmental processes. However, there were major disagreements in descriptions of comprehensive eutherian fibroblast growth factors gene data sets including either 18 or 22 homologues. The present analysis attempted to revise and update comprehensive eutherian fibroblast growth factor gene data sets, and address and resolve major discrepancies in their descriptions using eutherian comparative genomic analysis protocol and 35 public eutherian reference genomic sequence data sets.

Results: Among 577 potential coding sequences, the tests of reliability of eutherian public genomic sequences annotated most comprehensive curated eutherian third-party data gene data set of fibroblast growth factor genes including 267 complete coding sequences. The present study first described 8 superclusters including 22 eutherian fibroblast growth factor major gene clusters, proposing their updated classification and nomenclature.

Conclusions: The integrated gene annotations, phylogenetic analysis and protein molecular evolution analysis argued that comprehensive eutherian fibroblast growth factor gene data set classifications included 22 rather than 18 homologues.
\end{abstract}

Keywords: Gene annotations, Eutheria, Molecular evolution, Phylogenetic analysis, RRID:SCR_014401

\section{Background}

The eutherian fibroblast growth factors or FGFs were implicated as key developmental regulators [1-3]. First, the 15 paradigmatic paracrine or canonical fibroblast growth factors FGF1-10, FGF16-18, FGF20 and FGF22 were described as ligands to single-chain receptor tyrosine kinases named FGF receptors or FGFRs [2-11]. After paracrine FGF ligand and heparan sulphate glycosaminoglycan binding, the dimerized FGFRs become activated through autophosphorylation, interacting with cytosolic adaptor proteins and intracellular signaling cascades. Such transmembrane signal transduction was implicated in regulation of embryogenesis, implantation, gastrulation, body plan formation, branching morphogenesis and organogenesis, as well as in pathogeneses of

Correspondence: Marko.Premzl@alumni.anu.edu.au

The Australian National University Alumni, 4 Kninski trg Sq., Zagreb, Croatia human hereditary diseases including deafness, Kallmann syndrome, lacrimo-auriculo-dentodigital syndrome and different skeletal syndromes, and in tumorigenesis. Second, there were 3 endocrine fibroblast growth factors FGF19, FGF21 and FGF23 binding FGFRs and klotho protein cofactors $[2,3,7,12]$. The endocrine FGFs were implicated in metabolism regulation including phosphate and vitamin D homeostasis, cholesterol and bile acid homeostasis and glucose and lipid homeostasis, as well as in pathogenesis of autosomal dominant hypophosphataemic rickets. Third, the 4 intracellular fibroblast growth factors named fibroblast homologous factors included FGF11 or FHF3, FGF12 or FHF1, FGF13 or FHF2 and FGF14 or FGF4 [1, 3, 13-16]. The intracellular FGFs were described as regulators of nervous system development and function including integration and encoding of complex synaptic inputs into action potential outputs in central nervous system neurons, and

(c) The Author(s). 2020 Open Access This article is licensed under a Creative Commons Attribution 4.0 International License, which permits use, sharing, adaptation, distribution and reproduction in any medium or format, as long as you give appropriate credit to the original author(s) and the source, provide a link to the Creative Commons licence, and indicate if changes were made. The images or other third party material in this article are included in the article's Creative Commons licence, unless indicated otherwise in a credit line to the material. If material is not included in the article's Creative Commons licence and your intended use is not permitted by statutory regulation or exceeds the permitted use, you will need to obtain permission directly from the copyright holder. To view a copy of this licence, visit http://creativecommons.org/licenses/by/4.0/ The Creative Commons Public Domain Dedication waiver (http://creativecommons.org/publicdomain/zero/1.0/) applies to the data made available in this article, unless otherwise stated in a credit line to the data. 
implicated in pathogenesis of early-onset spinocerebellar ataxia. The molecular evolution and protein structure analyses indicated that eutherian FGFs folded into $\beta$ trefoil protein tertiary structures including 11 or $12 \beta$ strands $[1-3,7,12,13,17-28]$. However, there were major disagreements in descriptions of comprehensive eutherian FGF gene data sets. Specifically, Belov and Mohammadi [2] and Beenken and Mohammadi [7] argued that bona fide eutherian FGF homologues included 18 secreted paracrine and endocrine FGFs. On the other hand, the eutherian FGF classifications by Goldfarb [1] and Ornitz and Itoh [3] included both 18 secreted FGFs and 4 intracellular FGFs.

Undoubtedly, the public eutherian reference genomic sequence data sets advanced biological and medical sciences [29-34]. Indeed, the comparative genomics momentum was maintained by considerable international efforts in production and analysis of public eutherian reference genomic sequence data sets. For example, the initial sequencing and analysis of human genome attempted to revise and update human genes, and uncover potential new drugs, drug targets and molecular markers in medical diagnostics [35, 36]. Nevertheless, due to the incompleteness of eutherian reference genomic sequence assemblies $[35,37]$ and potential genomic sequence errors [36, 38], future updates and revisions of public eutherian reference genomic sequence data sets were expected. Inevitably, the potential genomic sequence errors including analytical and bioinformatical errors (erroneous gene annotations, genomic sequence misassemblies) and Sanger DNA sequencing method errors (artefactual nucleotide deletions, insertions and substitutions) could compromise unquestionable utility of public eutherian reference genomic sequence data sets. For example, Gajer et al. [39] described so-called lexicographical bias in some genomic sequence assemblers. In addition, the potential genomic sequence errors affecting phylogenetic analyses [40] were observed more frequently in reference genomic sequence assemblies including lower genomic sequence redundancies [41-43]. Thus, the eutherian comparative genomic analysis protocol was established as guidance in protection against potential genomic sequence errors in public eutherian reference genomic sequence data sets [44-46]. Using public eutherian reference genomic sequence data sets, the protocol published new test of reliability of public eutherian genomic sequences using genomic sequence redundancies, and new test of protein molecular evolution using relative synonymous codon usage statistics. The protocol revised and updated 12 eutherian gene data sets implicated in major physiological and pathological processes, including 1853 published complete coding sequences. Of note, there was positive correlation between genomic sequence redundancies of 35 public eutherian reference genomic sequence data sets respectively and published complete coding sequence numbers [46].

Therefore, the present analysis attempted to revise and update comprehensive eutherian FGF gene data sets, and address and resolve major disagreements in their descriptions using eutherian comparative genomic analysis protocol and 35 public eutherian reference genomic sequence data sets.

\section{Results}

\section{Gene annotations}

The tests of reliability of eutherian public genomic sequences annotated 267 FGF complete coding sequences among 577 FGF potential coding sequences (Fig. 1). The most comprehensive curated eutherian FGF third-party data gene data set was deposited in European Nucleotide Archive under accessions: LR130242-LR130508 [47, 48] (Additional file 1).

The present study first described 8 superclusters FGF1-8 including 22 major gene clusters of eutherian FGF genes, proposing their updated nomenclature (Fig. $1)$. The supercluster FGF1 included 4 major gene clusters FGF1A (11 FGF12 or FHF1 genes), FGF1B (9 FGF14 or FGF4 genes), FGF1C (11 FGF13 or FHF2 genes) and FGF1D (15 FGF11 or FHF3 genes) (Additional file 2AD). The supercluster $F G F 2$ included 2 major gene clusters FGF2A (8 FGF2 genes) and FGF2B (20 FGF1 genes) (Additional file 2E-F). The supercluster FGF3 included 1 major gene cluster FGF3A (17 FGF5 genes) (Additional file 2G). The supercluster FGF4 included 3 major gene clusters FGF4A (11 FGF2O genes), FGF4B (16 FGF9 genes) and FGF4C (14 FGF16 genes) (Additional file $2 \mathrm{H}-\mathrm{J})$. The supercluster $F G F 5$ included 4 major gene clusters FGF5A (14 FGF10 genes), FGF5B (16 FGF7 genes), FGF5C (7 FGF3 genes) and FGF5D (9 FGF22 genes) (Additional file $2 \mathrm{~K}-\mathrm{N}$ ). The supercluster $F G F 6$ included 3 major gene clusters FGF6A (5 FGF18 genes), FGF6B (12 FGF17 genes) and FGF6C (7 FGF8 genes) (Additional file 2O-Q). The supercluster $F G F 7$ included 2 major gene clusters FGF7A (8 FGF4 genes) and FGF7B (17 FGF6 genes) (Additional file 2R-S). Finally, The supercluster $F G F 8$ included 3 major gene clusters FGF8A (12 FGF19 genes), FGF8B (12 FGF23 genes) and FGF8C (16 FGF21 genes) (Additional file $2 \mathrm{~T}$-V).

The present study included new genomics tests of contiguity of eutherian public genomic sequences that analysed numbers of coding exons in FGF genes and their relative orientation (Additional files 1 and 2). The analysis including 903 FGF coding exons indicated that there were no coding exon misassemblies among 267 eutherian genomic sequences harbouring FGF complete coding sequences. The eutherian $F G F$ genes included either 5 coding exons (5 major gene clusters FGF1A-D 


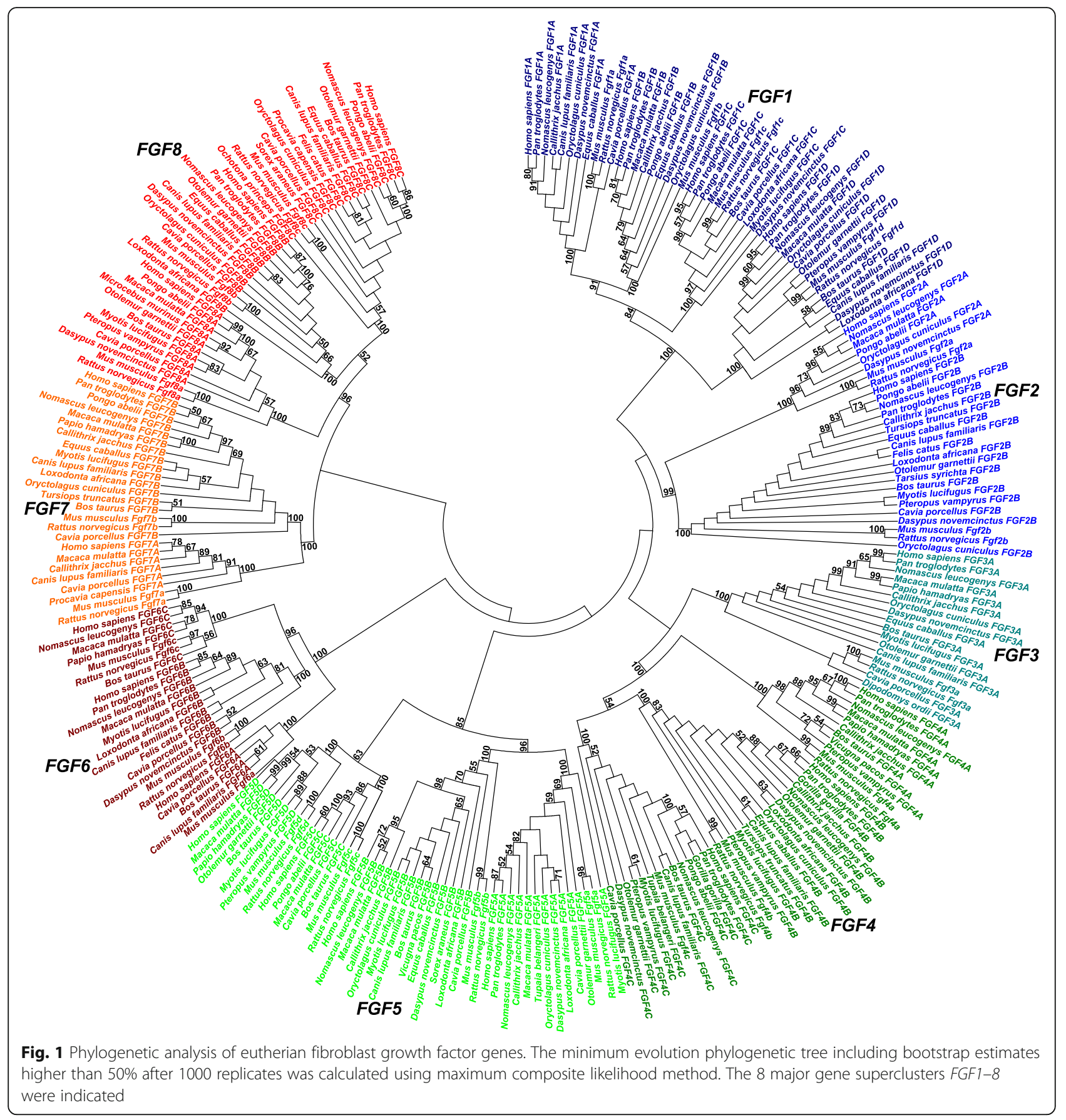

and FGF6A) or 3 coding exons (17 other major gene clusters). The eutherian FGF coding exon numbers were constant within major gene clusters, and there was no evidence of differential gene expansions indicating that 22 eutherian FGF major gene clusters respectively included orthologues. For example, whereas the human FGF1A gene included 5 coding exons along 264,215 bp (Additional file 2A), human FGF7A gene included 3 coding exons along $1776 \mathrm{bp}$ (Additional file $2 \mathrm{R}$ ).
Therefore, the present study annotating 22 eutherian FGF major gene clusters agreed with Goldfarb [1] and Ornitz and Itoh [3] but disagreed with Belov and Mohammadi [2] and Beenken and Mohammadi [7].

\section{Phylogenetic analysis}

The present minimum evolution phylogenetic tree calculations (Fig. 1) and calculations of pairwise nucleotide sequence identity patterns (Additional file 3) first classified 22 eutherian FGF major gene clusters among 8 
superclusters FGF1-8. The clustering of major gene clusters FGF1A-D within supercluster FGF1 agreed with subfamily FGF11 descriptions [3, 23], Smallwood et al. [13], Ornitz and Itoh [21], subfamily Fgf11/12/13/14 description [25] and Nam et al. [28]. The clustering of major gene clusters $F G F 2 A-B$ within supercluster $F G F 2$ agreed with subfamily FGF1 descriptions [3, 23], Smallwood et al. [13], Coulier et al. [17], Ornitz and Itoh [21], subfamily Fgf1/2 description [25] and Nam et al. [28]. The supercluster FGF3 description including 1 major gene cluster FGF3A agreed with Nam et al. [28] but disagreed with phylogenetic analyses of Ornitz and Itoh [3, 21], Coulier et al. [17] and Itoh and Ornitz [23, 25]. The clustering of major gene clusters $F G F 4 A-C$ within supercluster FGF4 agreed with subfamily FGF9 descriptions [3, 23], Ornitz and Itoh [21] and subfamily Fgf9/16/20 description [25] but disagreed with Nam et al. [28]. The clustering of major gene clusters $F G F 5 A-D$ within supercluster FGF5 disagreed with phylogenetic analyses of Ornitz and Itoh [3, 21], Itoh and Ornitz [23, 25] and Nam et al. [28]. The clustering of major gene clusters FGF6A-C within supercluster FGF6 agreed with subfamily FGF8 descriptions [3, 23], Ornitz and Itoh [21], subfamily Fgf8/17/18 description [25] and Nam et al. [28]. The clustering of major gene clusters $F G F 7 A-B$ within supercluster FGF7 agreed with Smallwood et al. [13], Coulier et al. [17], Ornitz and Itoh [21] and Nam et al. [28] but disagreed with Ornitz and Itoh [3] and Itoh and Ornitz [23, 25]. Finally, the clustering of major gene clusters FGF8A-C within supercluster FGF8 agreed with Ornitz and Itoh [21] but disagreed with Ornitz and Itoh [3], Itoh and Ornitz [23, 25] and Nam et al. [28].

Indeed, the calculations of pairwise nucleotide sequence identity patterns confirmed present phylogenetic classification of eutherian FGF genes (Additional file 3). The eutherian $F G F$ gene data set included average pairwise nucleotide sequence identity $\bar{a}=0,3 \quad\left(a_{\max }=1\right.$, $\left.a_{\min }=0,115, \bar{a}_{\mathrm{ad}}=0,094\right)[1-3,7,12,13,17,21,23,25-$ 28]. Among 22 eutherian FGF major gene clusters respectively, there were nucleotide sequence identity patterns of very close eutherian orthologues (FGF1A-B, $F G F 4 B$ ), close eutherian orthologues (FGF1C-D, FGF2A$B, F G F 4 A, F G F 4 C, F G F 5 B, F G F 6 A$, FGF7B), typical eutherian orthologues (FGF3A, FGF5A, FGF5C-D, FGF6B$C$, FGF7A, FGF8A, FGF8C) and distant eutherian orthologues $(F G F 8 B)$. In comparisons between eutherian $F G F$ major gene clusters within superclusters, there were nucleotide sequence identity patterns of very close eutherian homologues (superclusters FGF1-2, FGF4, FGF7), very close and close eutherian homologues (supercluster FGF6), close and typical eutherian homologues (supercluster FGF5) and typical eutherian homologues (supercluster FGF8). Finally, in comparisons between eutherian FGF major gene clusters between superclusters, there were nucleotide sequence identity patterns of close, typical, distant and very distant eutherian homologues.

Therefore, the present phylogenetic analysis proposed updated classification of eutherian $F G F$ genes.

\section{Protein molecular evolution analysis}

The protein molecular evolution analysis used protein primary structure features as major alignment landmarks in eutherian FGF protein amino acid sequence alignments, including common cysteine amino acid residues, common exon-intron splice site amino acid sites and common predicted $\mathrm{N}$-glycosylation sites (Fig. 2) (Additional file 4). There were between 1 and 9 common cysteine amino acid residues included among eutherian FGF major protein clusters respectively. For example, whereas the major protein cluster FGF5D included 1 common cysteine amino acid residue, major protein cluster FGF5A included 9 common cysteine amino acid residues. There were either 4 common exon-intron splice site amino acid sites (5 major protein clusters FGF1A-D and FGF6A) or 2 common exon-intron splice site amino acid sites (17 other major protein clusters) among eutherian FGF major protein clusters respectively. Finally, there were between 0 and 2 common predicted N-glycosylation sites among eutherian FGF major protein clusters respectively.

Next, the tests of protein molecular evolution first calculated relative synonymous codon usage statistics $(R)$ of eutherian FGF gene data set using 267 FGF complete coding sequences (Additional file 4), and described 20 amino acid codons including $R \leq 0,7$ as not preferable amino acid codons (Fig. 3a). The tests used human FGF1A protein primary structure as reference protein amino acid sequence (Fig. 3b). Among 243 human FGF1A protein amino acid residues, the tests of protein molecular evolution described 19 invariant amino acid sites, viz.: M1, C41, C55, P68, Q69, L70, K71, G72, I73, V74, T75, L77, G112, M129, G133, C145, Y159, G181 and C206, as well as 3 forward amino acid sites S101, E149 and Y208. First, the human FGF1A amino acid sites M1, L77, G133, C145 and Y159 were invariant among 267 eutherian FGF protein primary structures (except that M1 was invariant among 266 FGF protein primary structures). For example, the human FGF1A invariant amino acid sites L77, G133 and C145 were described by Goetz et al. [12, 24], Smallwood et al. [13], Coulier et al. [17], Venkataraman et al. [18], Plotnikov et al. [19] and Olsen et al. [22]. Furthermore, the human FGF1A amino acid sites G112 and M129 respectively were invariant among 21 eutherian FGF major protein clusters. For example, the human FGF1A amino acid site G112 was homologous to human FGF2B amino amino acid site $\mathrm{G} 67$ that was implicated in interactions between FGF2B ligand and FGFR2 receptor [19, 20]. In addition, 


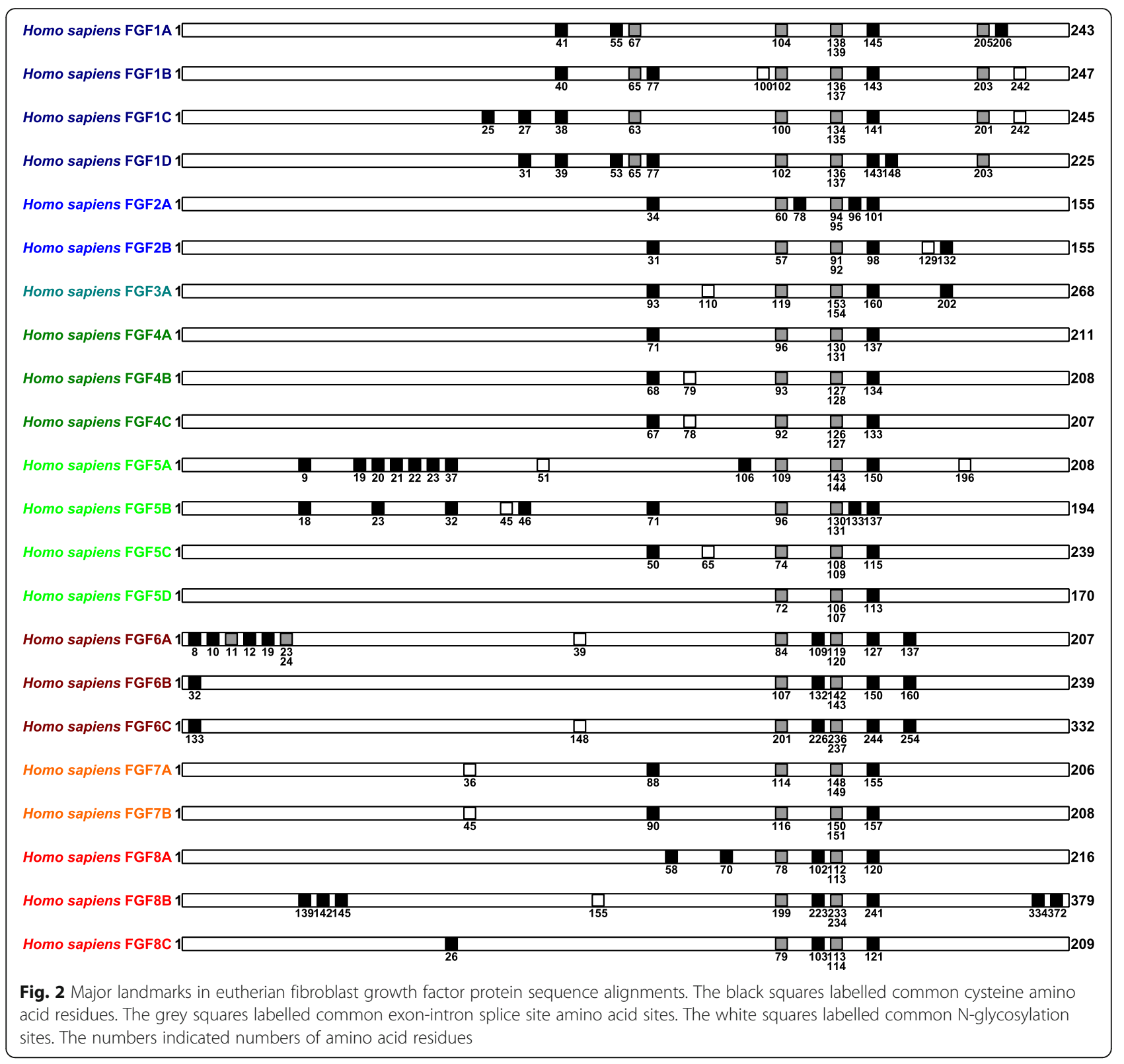

the human FGF1A amino acid site G181 that was invariant among 7 eutherian FGF1-7 protein superclusters was described as first glycine amino acid residue in paracrine FGF glycine box protein amino acid sequence motif G-x(4)-G-x(2)-S/T [2]. The human FGF1A amino acid sites P68, Q69, L70, K71, G72, I73, V74 and T75 were invariant among 4 eutherian FGF1A-D major protein clusters. For example, the human FGF1A amino acid sites $\mathrm{K} 71$ and I73 were described as residues engaged in voltage-gated sodium channel binding [24]. Finally, the human FGF1A forward amino acid sites S101 and E149 were described among 267 eutherian FGF protein primary structures, and forward amino acid site Y208 was described among 2 eutherian FGF1-2 protein superclusters. For example, the human FGF1A forward amino acid site E149 was homologous to human FGF2A amino amino acid site E105 that was implicated in hydrogen bonding between FGF2A ligand and D3 domain of FGFR2 receptor $[19,26]$.

Therefore, the tests of protein molecular evolution using relative synonymous codon usage statistics described amino acid sites implicated as critical in FGF protein secondary, tertiary and quaternary structural features.

\section{Discussion}

The major disagreements in descriptions of comprehensive eutherian FGF gene data sets included classifications 


\begin{tabular}{|c|c|c|c|c|c|c|c|c|c|c|c|}
\hline \multicolumn{12}{|l|}{$\mathbf{A}$} \\
\hline Codon & Counts & $R$ & Codon & Counts & $\boldsymbol{R}$ & Codon & Counts & $R$ & Codon & Counts & $R$ \\
\hline TTT (F) & 890 & 0,71 & TCT (S) & 656 & 0,79 & TAT (Y) & 750 & 0,65 & TGT (C) & 347 & 0,64 \\
\hline TTC (F) & 1607 & 1,29 & TCC (S) & 1109 & 1,34 & TAC $(Y)$ & 1546 & 1,35 & TGC (C) & 735 & 1,36 \\
\hline TTA (L) & 325 & 0,34 & TCA (S) & 489 & 0,59 & TAA (\&) & 86 & - & TGA (\&) & 95 & - \\
\hline TTG (L) & 659 & 0,69 & TCG (S) & 403 & 0,49 & TAG (\&) & 86 & - & TGG (W) & 606 & 1 \\
\hline Codon & Counts & $R$ & Codon & Counts & $R$ & Codon & Counts & $R$ & Codon & Counts & $\boldsymbol{R}$ \\
\hline CTT (L) & 435 & 0,46 & CCT (P) & 712 & 0,81 & CAT (H) & 418 & 0,48 & CGT (R) & 253 & 0,32 \\
\hline CTC (L) & 1406 & 1,48 & $\operatorname{ccc}(\mathrm{P})$ & 1486 & 1,69 & CAC (H) & 1333 & 1,52 & CGC (R) & 1158 & 1,47 \\
\hline CTA (L) & 349 & 0,37 & CCA (P) & 749 & 0,85 & CAA (Q) & 527 & 0,46 & CGA (R) & 465 & 0,59 \\
\hline CTG (L) & 2528 & 2,66 & $\operatorname{CCG}(\mathrm{P})$ & 568 & 0,65 & CAG (Q) & 1765 & 1,54 & CGG (R) & 1174 & 1,49 \\
\hline Codon & Counts & $\boldsymbol{R}$ & Codon & Counts & $R$ & Codon & Counts & $R$ & Codon & Counts & $\boldsymbol{R}$ \\
\hline ATT (I) & 496 & 0,72 & АCT (T) & 523 & 0,72 & AAT (N) & 744 & 0,71 & AGT (S) & 667 & 0,8 \\
\hline ATC (I) & 1213 & 1,77 & $\mathrm{ACC}(\mathrm{T})$ & 1204 & 1,67 & $A A C(N)$ & 1352 & 1,29 & AGC (S) & 1650 & 1,99 \\
\hline ATA (I) & 350 & 0,51 & $\mathrm{ACA}(\mathrm{T})$ & 667 & 0,92 & AAA (K) & 1410 & 0,82 & AGA (R) & 687 & 0,87 \\
\hline ATG (M) & 1207 & 1 & ACG (T) & 492 & 0,68 & AAG (K) & 2023 & 1,18 & AGG (R) & 993 & 1,26 \\
\hline Codon & Counts & $\boldsymbol{R}$ & Codon & Counts & $\boldsymbol{R}$ & Codon & Counts & $R$ & Codon & Counts & $\boldsymbol{R}$ \\
\hline GTT (V) & 415 & 0,49 & GCT (A) & 653 & 0,74 & GAT (D) & 657 & 0,69 & GGT (G) & 487 & 0,38 \\
\hline GTC (V) & 798 & 0,94 & GCC (A) & 1666 & 1,88 & GAC (D) & 1261 & 1,31 & GGC (G) & 2115 & 1,65 \\
\hline GTA (V) & 280 & 0,33 & GCA (A) & 642 & 0,72 & GAA (E) & 1268 & 0,81 & GGA (G) & 1155 & 0,9 \\
\hline GTG (V) & 1912 & 2,25 & GCG (A) & 584 & 0,66 & GAG (E) & 1865 & 1,19 & GGG (G) & 1382 & 1,08 \\
\hline \multicolumn{12}{|l|}{$\mathbf{B}$} \\
\hline \multicolumn{12}{|c|}{ Homo sapiens FGF1A } \\
\hline \multicolumn{2}{|c|}{ Homo sapiens FGF1A } & \multicolumn{10}{|c|}{ 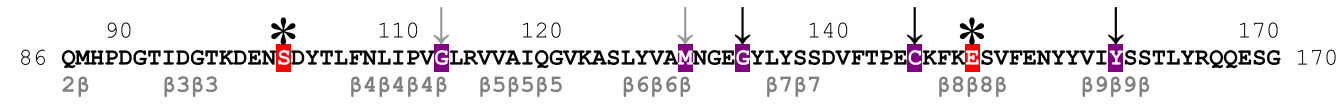 } \\
\hline \multicolumn{12}{|c|}{ 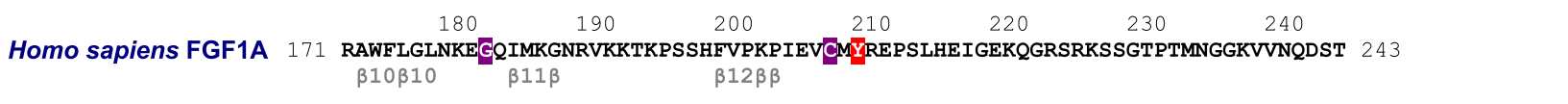 } \\
\hline \multicolumn{12}{|c|}{$\begin{array}{l}\text { Fig. } 3 \text { Tests of protein molecular evolution of eutherian fibroblast growth factors. a Relative synonymous codon usage statistics of eutherian FGF } \\
\text { gene data set. The not preferable amino acid codons were indicated by white letters on red backgrounds. Counts, observed amino acid codon } \\
\text { counts; } R \text {, relative synonymous codon usage statistics; \&, stop codons. b Reference human FGF1A protein amino acid sequence. The } 19 \text { invariant } \\
\text { amino acid sites were shown using white letters on violet backgrounds. Whereas the } 5 \text { amino acid sites that were invariant among } 22 \text { FGF major } \\
\text { protein clusters were indicated by black arrows (except that M1 was invariant among } 266 \text { FGF protein primary structures), grey arrows indicated } 2 \\
\text { amino acid sites that were invariant among } 21 \text { FGF major protein clusters respectively. The } 3 \text { forward amino acid sites were shown using white } \\
\text { letters on red backgrounds. The stars labelled } 2 \text { forward amino acid sites described among } 22 \text { FGF major protein clusters. The positions of } 12 \beta- \\
\text { strands implicated in } \beta \text {-trefoil protein tertiary structure were indicated below reference human FGF1A protein primary structure [22, 24] }\end{array}$} \\
\hline
\end{tabular}

of either 18 FGF genes [2, 7] or 22 FGF genes [1, 3]. The present analysis attempted to address and resolve these discrepancies using eutherian comparative genomic analysis protocol and public eutherian reference genomic sequence data sets [29-36, 44-46]. The advantages of eutherian reference genomic sequence data sets were well established phylogeny [29, 30, 34] and calibrated taxon sampling including genomic sequence redundancies that were applicable in tests of reliability of eutherian public genomic sequences [31-33]. Therefore, the tests of reliability of eutherian public genomic sequences annotated most comprehensive curated eutherian third- party data gene data set of FGF genes that included 267 complete coding sequences among 577 potential coding sequences. Second, the present study first described 8 superclusters of eutherian FGF genes that included 22 major gene clusters, proposing their updated nomenclature. Third, the new genomics tests of contiguity of eutherian public genomic sequences included 903 coding exons, and annotated either 3 or 5 coding exons in eutherian $F G F$ genes including no evidence of differential gene expansions. Fourth, the present phylogenetic analysis proposed updated classification of eutherian $F G F$ genes. Finally, the tests of protein molecular evolution 
using relative synonymous codon usage statistics described 19 invariant amino acid sites and 3 forward amino acid sites in reference human FGF1A protein primary structure, including amino acid residues described as critical in FGF protein secondary, tertiary and quaternary structural features. In conclusion, the present comparative genomic analysis integrating gene annotations, phylogenetic analysis and protein molecular evolution analysis argued that $22 F G F$ genes [1, 3], rather than 18 FGF genes [2, 7], were included in comprehensive eutherian FGF gene data set classifications.

\section{Methods}

\section{Eutherian comparative genomic analysis protocol}

The eutherian comparative genomic analysis protocol RRID:SCR_014401 integrated gene annotations, phylogenetic analysis and protein molecular evolution analysis with tests of reliability of eutherian public genomic sequences, tests of contiguity of eutherian public genomic sequences and tests of protein molecular evolution into one framework of eutherian gene descriptions (Fig. 4) [44-46].

\section{Gene annotations}

The protocol used gene identifications in 35 public genomic sequence assemblies, tests of reliability of eutherian public genomic sequences and new genomics tests of contiguity of eutherian public genomic sequences in eutherian FGF gene annotations. First, the sequence alignment editor BioEdit 7.0.5.3 was used in all analyses and manipulations of nucleotide and protein sequences [49]. The National Center for Biotechnology Information (NCBI) BLAST Genomes was used in identifications of FGF potential coding sequences in eutherian reference genomic sequence data sets [50-53], as well as Ensembl genome browser BLAST or BLAT tools $[54,55]$. Second,

\title{
EUTHERIAN COMPARATIVE GENOMIC ANALYSIS PROTOCOL GENE ANNOTATIONS \\ GENE IDENTIFICATIONS \\ ANALYSES OF GENE FEATURES
}

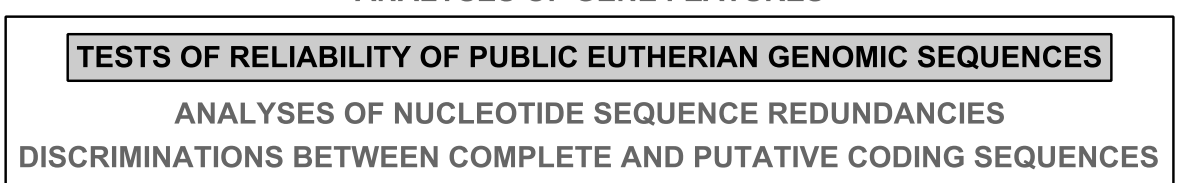

TESTS OF CONTIGUITY OF PUBLIC EUTHERIAN GENOMIC SEQUENCES
MULTIPLE PAIRWISE GENOMIC SEQUENCE ALIGNMENTS
ANALYSES OF CODING EXON NUMBERS AND THEIR RELATIVE ORIENTATIONS

CURATED EUTHERIAN THIRD PARTY DATA GENE DATA SETS

\author{
$\downarrow$ \\ PHYLOGENETIC ANALYSIS \\ PHYLOGENETIC TREE CALCULATIONS \\ ANALYSES OF DIFFERENTIAL GENE EXPANSIONS
}

ALIGNMENTS OF PROTEIN AND NUCLEOTIDE SEQUENCES

PAIRWISE NUCLEOTIDE SEQUENCE IDENTITY CALCULATIONS

PROTEIN MOLECULAR EVOLUTION ANALYSIS

PROTEIN AMINO ACID SEQUENCE FEATURE ANALYSES

TESTS OF PROTEIN MOLECULAR EVOLUTION

RELATIVE SYNONYMOUS CODON USAGE STATISTIC CALCULATIONS INVARIANT, FORWARD AND COMPENSATORY AMINO ACID SITE DESCRIPTIONS

Fig. 4 Eutherian comparative genomic analysis protocol flowchart 
the tests of reliability of eutherian public genomic sequences used FGF potential coding sequences. Using BLASTN and primary Sanger DNA sequencing information deposited in NCBI Trace Archive [51, 56], the first test steps analysed nucleotide sequence coverages of each $F G F$ potential coding sequence. If consensus trace sequence coverages were available for every nucleotide, the protocol described $F G F$ potential coding sequences as FGF complete coding sequences. However, if consensus trace sequence coverages were not available for every nucleotide, the protocol described FGF potential coding sequences as FGF putative coding sequences (not used in analyses). The protocol then deposited FGF complete coding sequences in European Nucleotide Archive as curated third-party data gene information [57-60]. The protocol used guidelines of human gene nomenclature [61] and guidelines of mouse gene nomenclature [62] in updated eutherian FGF gene classification and nomenclature. Third, the protocol used new genomics tests of contiguity of eutherian public genomic sequences in eutherian FGF gene annotations. Using multiple pairwise genomic sequence alignments of eutherian genomic sequences harbouring FGF complete coding sequences, the tests of contiguity analysed numbers of coding exons in FGF genes and their relative orientation. The tests discriminated between FGF genes not including coding exon misassemblies in eutherian genomic sequence assemblies and FGF genes including coding exon misassemblies. The tests used mVISTA AVID option in multiple pairwise genomic sequence alignments, using default settings $[63,64]$. The empirically determined cut-offs of detection of common genomic sequence regions in pairwise alignments with base sequences (Homo sapiens) were 95\% nucleotide sequence identity along $100 \mathrm{bp}$ (Pan troglodytes, Gorilla gorilla), 90\% along 100 bp (Pongo abelii, Nomascus leucogenys), 85\% along 100 bp (Macaca mulatta, Papio hamadryas), 80\% along 100 bp (Callithrix jacchus), 75\% along 100 bp (Tarsius syrichta, Microcebus murinus, Otolemur garnettii), 65\% along 100 bp (Rodentia) or 70\% along 100 bp in other pairwise alignments [44-46]. In preparatory steps of multiple pairwise genomic sequence alignments, the protocol did not include masking of transposable elements in genomic sequences harbouring FGF complete coding sequences.

\section{Phylogenetic analysis}

The protocol used protein and nucleotide sequence alignments, calculations of phylogenetic trees, calculations of pairwise nucleotide sequence identities and analysis of differential gene expansions in phylogenetic analysis of eutherian FGF gene data set. First, using BioEdit 7.0.5.3, the protocol translated FGF complete coding sequences, and aligned them at amino acid level using ClustalW implemented in BioEdit 7.0.5.3. After manual corrections of FGF protein primary structure alignments, the $F G F$ nucleotide sequence alignments were prepared accordingly. Second, the MEGA 6.06 program was used in phylogenetic tree calculations, using minimum evolution method that was applicable in phylogenetic analysis of very close, close, typical, distant and very distant eutherian FGF homologues (default settings, except gaps $/$ missing data treatment $=$ pairwise deletion and maximum composite likelihood method) [65, 66]. Third, the protocol used BioEdit 7.0.5.3 in calculations of pairwise nucleotide sequence identities of $F G F$ complete coding sequences that were used in statistical analyses. The Microsoft Office Excel common statistical functions were used in calculations of pairwise nucleotide sequence identity patterns of eutherian FGF gene data set. Using pairwise nucleotide sequence identities of FGF nucleotide sequence alignments including 267 FGF complete coding sequences, the protocol calculated average pairwise nucleotide sequence identities $(\bar{a})$ and their average absolute deviations $\left(\bar{a}_{\mathrm{ad}}\right)$, and largest $\left(a_{\mathrm{max}}\right)$ and smallest $\left(a_{\mathrm{min}}\right)$ pairwise nucleotide sequence identities.

\section{Protein molecular evolution analysis}

The protocol used analysis of FGF protein amino acid sequence features and tests of protein molecular evolution integrating patterns of $F G F$ nucleotide sequence similarities with FGF protein primary structures in protein molecular evolution analysis. The protocol used complete $F G F$ nucleotide sequence alignments in tests of protein molecular evolution, including 267 FGF complete coding sequences and 58,533 codons. Among eutherian FGF complete coding sequences, the average number of codons was 219. Using MEGA 6.06, the relative synonymous codon usage statistics were calculated as ratios between observed and expected amino acid codon counts $(R=$ Counts / Expected counts). The protocol then described 20 amino acid codons including $R \leq 0,7$ as not preferable amino acid codons, viz.: TTA, TTG, CTT, CTA, ATA, GTT, GTA, TCA, TCG, CCG, ACG, GCG, TAT, CAT, CAA, GAT, TGT, CGT, CGA, GGT (Fig. 3b). Finally, the protocol described reference human FGF1A protein sequence amino acid sites as invariant amino acid sites (invariant alignment positions), forward amino acid sites (variant alignment positions that did not include amino acid codons with $R \leq 0,7$ ) or compensatory amino acid sites (variant alignment positions that included amino acid codons with $R \leq 0,7$ ).

\section{Supplementary information}

Supplementary information accompanies this paper at https://doi.org/10. 1186/s12864-020-06958-4.

Additional file 1. Third-party data gene data set of eutherian fibroblast growth factor genes. 
Additional file 2 Multiple pairwise genomic sequence alignments of eutherian fibroblast growth factor genes. The FGF coding exon sequence regions in base sequences (Homo sapiens) were displayed as indigo rectangles, and grey arrows indicated their relative orientation (top). The genomic sequence regions including sequence identity levels above empirical cut-offs of detection of common genomic sequence regions were shown accordingly in multiple pairwise alignments.

Additional file 3. Pairwise nucleotide sequence identity patterns of eutherian fibroblast growth factor genes.

Additional file 4. Protein amino acid sequence alignments of eutherian fibroblast growth factors. The amino acid positions were labelled using white letters on black background (100\% sequence identity level), white letters on dark grey background ( $\geq 75 \%$ sequence identity level) or black letters on grey background ( $\geq 50 \%$ sequence identity level). The 19 invariant amino acid sites were shown using white letters on violet backgrounds and 3 forward amino acid sites were shown using white letters on red backgrounds in reference human FGF1A protein primary structure (top). The stop codons were indicated by \&s.

\section{Abbreviations}

FGF: Fibroblast growth factor; FGF1-8: Eutherian fibroblast growth factor gene superclusters

\section{Acknowledgements}

MP would like to thank manuscript reviewers on their comments and suggestions.

\section{Author's contributions}

MP conceived and conducted experiments, and prepared manuscript. The author read and approved the final manuscript.

\section{Funding}

Not applicable.

\section{Availability of data and materials}

The original curated third-party data gene data set including 267 eutherian FGF complete coding sequences was deposited in European Nucleotide Archive under accessions: LR130242-LR130508 [47]. The present study was registered under NCBI BioProject entitled "Curated eutherian third-party data gene data sets" (NCBI BioProject accession: PRJNA453891; NCBI BioSample accessions: SAMN09005565-SAMN09005599) [48, 67]. The public eutherian reference genomic sequence data sets (Additional file 1) were available in NCBI GenBank [51, 52] and Ensembl [54].

\section{Ethics approval and consent to participate}

Not applicable.

\section{Consent for publication}

Not applicable.

\section{Competing interests}

No competing interests were declared.

\section{Received: 29 November 2019 Accepted: 29 July 2020}

Published online: 05 August 2020

\section{References}

1. Goldfarb M. Fibroblast growth factor homologous factors: evolution, structure, and function. Cytokine Growth Factor Rev. 2005;16:215-20.

2. Belov AA, Mohammadi M. Molecular mechanisms of fibroblast growth factor signaling in physiology and pathology. Cold Spring Harb Perspect Biol. 2013;5:a015958.

3. Ornitz DM, Itoh N. The fibroblast growth factor signaling pathway. Wiley Interdiscip Rev Dev Biol. 2015;4:215-66.

4. Martin GR. The roles of FGFs in the early development of vertebrate limbs. Genes Dev. 1998;12:1571-86.

5. Hogan BL. Morphogenesis. Cell. 1999;96:225-33.

6. Liu JP, Laufer E, Jessell TM. Assigning the positional identity of spinal motor neurons: rostrocaudal patterning of Hox-c expression by FGFs, Gdf11, and retinoids. Neuron. 2001;32:997-1012.
7. Beenken A, Mohammadi M. The FGF family: biology, pathophysiology and therapy. Nat Rev Drug Discov. 2009;8:235-53.

8. Makarenkova HP, Hoffman MP, Beenken A, Eliseenkova AV, Meech R, Tsau C, et al. Differential interactions of FGFs with heparan sulfate control gradient formation and branching morphogenesis. Sci Signal. 2009;2:ra55.

9. Ornitz DM, Marie PJ. Fibroblast growth factor signaling in skeletal development and disease. Genes Dev. 2015;29:1463-86.

10. Brewer JR, Mazot P, Soriano P. Genetic insights into the mechanisms of Fgf signaling. Genes Dev. 2016;30:751-71.

11. Patel VN, Pineda DL, Hoffman MP. The function of heparan sulfate during branching morphogenesis. Matrix Biol. 2017;57-58:311-23.

12. Goetz R, Beenken A, Ibrahimi OA, Kalinina J, Olsen SK, Eliseenkova AV, et al. Molecular insights into the klotho-dependent, endocrine mode of action of fibroblast growth factor 19 subfamily members. Mol Cell Biol. 2007;27:341728.

13. Smallwood PM, Munoz-Sanjuan I, Tong P, Macke JP, Hendry SH, Gilbert DJ, et al. Fibroblast growth factor (FGF) homologous factors: new members of the FGF family implicated in nervous system development. Proc Natl Acad Sci U S A. 1996;93:9850-7.

14. Goldfarb M. Signaling by fibroblast growth factors: the inside story. Sci STKE. 2001;2001:pe37.

15. Schoorlemmer J, Goldfarb M. Fibroblast growth factor homologous factors are intracellular signaling proteins. Curr Biol. 2001;11:793-7.

16. Goldfarb M, Schoorlemmer J, Williams A, Diwakar S, Wang Q, Huang X, et al. Fibroblast growth factor homologous factors control neuronal excitability through modulation of voltage-gated sodium channels. Neuron. 2007;55: 449-63.

17. Coulier F, Pontarotti P, Roubin R, Hartung H, Goldfarb M, Birnbaum D. Of worms and men: an evolutionary perspective on the fibroblast growth factor (FGF) and FGF receptor families. J Mol Evol. 1997;44:43-56.

18. Venkataraman G, Raman R, Sasisekharan V, Sasisekharan R. Molecular characteristics of fibroblast growth factor-fibroblast growth factor receptorheparin-like glycosaminoglycan complex. Proc Natl Acad Sci U S A. 1999;96: 3658-63.

19. Plotnikov AN, Hubbard SR, Schlessinger J, Mohammadi M. Crystal structures of two FGF-FGFR complexes reveal the determinants of ligand-receptor specificity. Cell. 2000;101:413-24.

20. Stauber DJ, DiGabriele AD, Hendrickson WA. Structural interactions of fibroblast growth factor receptor with its ligands. Proc Natl Acad Sci U S A. 2000;97:49-54

21. Ornitz DM, Itoh N. Fibroblast growth factors. Genome Biol. 2001;2: Reviews3005.

22. Olsen SK, Garbi M, Zampieri N, Eliseenkova AV, Ornitz DM, Goldfarb M, et al. Fibroblast growth factor (FGF) homologous factors share structural but not functional homology with FGFs. J Biol Chem. 2003;278:34226-36.

23. Itoh N, Ornitz DM. Evolution of the Fgf and Fgfr gene families. Trends Genet. 2004;20:563-9.

24. Goetz R, Dover K, Laezza F, Shtraizent N, Huang X, Tchetchik D, et al. Crystal structure of a fibroblast growth factor homologous factor (FHF) defines a conserved surface on FHFs for binding and modulation of voltage-gated sodium channels. J Biol Chem. 2009;284:17883-96.

25. Itoh N, Ornitz DM. Fibroblast growth factors: from molecular evolution to roles in development, metabolism and disease. J Biochem. 2011;149:121-30.

26. Goetz R, Mohammadi M. Exploring mechanisms of FGF signalling through the lens of structural biology. Nat Rev Mol Cell Biol. 2013;14:166-80.

27. Bertrand S, Iwema T, Escriva H. FGF signaling emerged concomitantly with the origin of Eumetazoans. Mol Biol Evol. 2014;31:310-8.

28. Nam K, Lee KW, Chung O, Yim HS, Cha SS, Lee SW, et al. Analysis of the FGF gene family provides insights into aquatic adaptation in cetaceans. Sci Rep. 2017;7:40233.

29. Murphy WJ, Eizirik E, Johnson WE, Zhang YP, Ryder OA, O'Brien SJ. Molecular phylogenetics and the origins of placental mammals. Nature. 2001:409:614-8.

30. Wilson DE, Reeder DM. Mammal species of the world: a taxonomic and geographic reference. 3rd ed. Baltimore: The Johns Hopkins University Press; 2005

31. Blakesley RW, Hansen NF, Mullikin JC, Thomas PJ, McDowell JC, Maskeri B, et al. An intermediate grade of finished genomic sequence suitable for comparative analyses. Genome Res. 2004;14:2235-44.

32. Margulies EH, Vinson JP, Miller W, Jaffe DB, Lindblad-Toh $\mathrm{K}$, Chang JL, et al. An initial strategy for the systematic identification of functional elements in 
the human genome by low-redundancy comparative sequencing. Proc Natl Acad Sci U S A. 2005;102:4795-800.

33. Lindblad-Toh K, Garber M, Zuk O, Lin MF, Parker BJ, Washietl S, et al. A highresolution map of human evolutionary constraint using 29 mammals. Nature. 2011;478:476-82.

34. O'Leary MA, Bloch Jl, Flynn JJ, Gaudin TJ, Giallombardo A, Giannini NP, et al. The placental mammal ancestor and the post-K-Pg radiation of placentals. Science. 2013;339:662-7.

35. International Human Genome Sequencing Consortium. Initial sequencing and analysis of the human genome. Nature. 2001;409:860-921.

36. International Human Genome Sequencing Consortium. Finishing the euchromatic sequence of the human genome. Nature. 2004;431:931-45.

37. Frankish A, Diekhans M, Ferreira AM, Johnson R, Jungreis I, Loveland J, et al. GENCODE reference annotation for the human and mouse genomes. Nucleic Acids Res. 2019;47:D766-73.

38. Mouse Genome Sequencing Consortium. Lineage-specific biology revealed by a finished genome assembly of the mouse. PLoS Biol. 2009;7:1000112.

39. Gajer P, Schatz M, Salzberg SL. Automated correction of genome sequence errors. Nucleic Acids Res. 2004;32:562-9.

40. Di Franco A, Poujol R, Baurain D, Philippe H. Evaluating the usefulness of alignment filtering methods to reduce the impact of errors on evolutionary inferences. BMC Evol Biol. 2019;19:21.

41. Hubisz MJ, Lin MF, Kellis M, Siepel A. Error and error mitigation in lowcoverage genome assemblies. PLoS One. 2011;6:e17034.

42. Prosdocimi F, Linard B, Pontarotti P, Poch O, Thompson JD. Controversies in modern evolutionary biology: the imperative for error detection and quality control. BMC Genomics. 2012;13:5.

43. Denton JF, Lugo-Martinez J, Tucker AE, Schrider DR, Warren WC, Hahn MW. Extensive error in the number of genes inferred from draft genome assemblies. PLoS Comput Biol. 2014;10:e1003998.

44. Premzl M. Eutherian comparative genomic analysis protocol. Nat Protoc. 2018. https://doi.org/10.1038/protex.2018.028.

45. Premzl M. Comparative genomic analysis of eutherian connexin genes. Sci Rep. 2019;9:16938.

46. Premzl M. Eutherian third-party data gene collections. Gene Rep. 2019;16: 100414.

47. European Nucleotide Archive. Accessions: LR130242-LR130508. https://www. ebi.ac.uk/ena/data/view/LR130242-LR130508..

48. NCBI BioProject. Accession: PRJNA453891. https://www.ncbi.nlm.nih.gov/ bioproject/453891. Accessed 27 Jul 2020.

49. BioEdit. https://bioedit.software.informer.com/. Accessed 27 Jul 2020.

50. Camacho C, Coulouris G, Avagyan V, Ma N, Papadopoulos J, Bealer K, et al. BLAST+: architecture and applications. BMC Bioinformatics. 2009;10:421.

51. Sayers EW, Beck J, Brister JR, Bolton EE, Canese K, Comeau DC, et al. Database resources of the National Center for biotechnology information. Nucleic Acids Res. 2020;48:D9-16.

52. Sayers EW, Cavanaugh M, Clark K, Ostell J, Pruitt KD, Karsch-Mizrachi I. GenBank. Nucleic Acids Res. 2020:48:D84-6.

53. NCBI BLAST Genomes. https://blast.ncbi.nlm.nih.gov/Blast.cgi. Accessed 27 Jul 2020.

54. Yates AD, Achuthan P, Akanni W, Allen J, Allen J, Alvarez-Jarreta J, et al. Ensembl 2020. Nucleic Acids Res. 2020;48:D682-8.

55. Ensembl genome browser. https://www.ensembl.org. Accessed 27 Jul 2020.

56. NCBI Trace Archive. https://www.ncbi.nlm.nih.gov/Traces/trace.cgi. Accessed 27 Jul 2020

57. Gibson R, Alako B, Amid C, Cerdeno-Tarraga A, Cleland I, Goodgame N, et al. Biocuration of functional annotation at the European nucleotide archive. Nucleic Acids Res. 2016;44:D58-66.

58. Karsch-Mizrachi I, Takagi T, Cochrane G. The international nucleotide sequence database collaboration. Nucleic Acids Res. 2018;46:D48-51.

59. Amid C, Alako BTF, Balavenkataraman Kadhirvelu V, Burdett T, Burgin J, Fan J, et al. The European nucleotide archive in 2019. Nucleic Acids Res. 2020;48: D70-6.

60. European Nucleotide Archive. https://www.ebi.ac.uk/ena/about/tpa-policy. Accessed 27 Jul 2020.

61. Guidelines of human gene nomenclature. http://www.genenames.org/ about/guidelines. Accessed $27 \mathrm{Jul} 2020$

62. Guidelines of mouse gene nomenclature. http://www.informatics.jax.org/ mgihome/nomen/gene.shtml. Accessed 27 Jul 2020.
63. Poliakov A, Foong J, Brudno M, Dubchak I. GenomeVISTA--an integrated software package for whole-genome alignment and visualization. Bioinformatics. 2014;30:2654-5.

64. mVISTA. http://genome.lbl.gov/vista/index.shtml. Accessed 27 Jul 2020.

65. Kumar S, Stecher G, Li M, Knyaz C, Tamura K. MEGA X: Molecular evolutionary genetics analysis across computing platforms. Mol Biol Evol. 2018;35:1547-9.

66. MEGA 6.06. http://www.megasoftware.net/. Accessed 27 Jul 2020.

67. Barrett T, Clark K, Gevorgyan R, Gorelenkov V, Gribov E, Karsch-Mizrachi I, et al. BioProject and BioSample databases at NCBI: facilitating capture and organization of metadata. Nucleic Acids Res. 2012;40:D57-63.

\section{Publisher's Note}

Springer Nature remains neutral with regard to jurisdictional claims in published maps and institutional affiliations.
Ready to submit your research? Choose BMC and benefit from:

- fast, convenient online submission

- thorough peer review by experienced researchers in your field

- rapid publication on acceptance

- support for research data, including large and complex data types

- gold Open Access which fosters wider collaboration and increased citations

- maximum visibility for your research: over $100 \mathrm{M}$ website views per year

At BMC, research is always in progress.

Learn more biomedcentral.com/submissions 